\title{
Comparison of biological characteristics of nucleus pulposus mesenchymal stem cells derived from non-degenerative and degenerative human nucleus pulposus
}

\author{
ZHIWEI JIA $^{1,2^{*}}$, PUSHAN YANG ${ }^{3 *}$, YAOHONG WU $^{1 *}$, YONG TANG $^{1}$, YACHAO ZHAO $^{1}$, \\ JIANHONG WU ${ }^{4}$, DELI WANG ${ }^{1}$, QING HE ${ }^{1}$ and DIKE RUAN ${ }^{1}$ \\ ${ }^{1}$ Department of Orthopaedics, Navy General Hospital, Beijing 100048; ${ }^{2}$ Department of Orthopaedics, \\ Clinical Center in Beijing Space, The 306th Hospital of People's Liberation Army, Beijing 100094; \\ ${ }^{3}$ Department of Orthopaedics, Guangyuan Central Hospital, Guangyuan, Sichuan 628000; \\ ${ }^{4}$ Department of Orthopaedics, Shanghai General Hospital, Shanghai 200080, P.R. China
}

Received January 5, 2016; Accepted January 6, 2017

DOI: $10.3892 / e t m .2017 .4398$

\begin{abstract}
Cell therapy using mesenchymal stem cells provides a promising approach for the treatment of intervertebral disc degeneration (IDD). In recent years, human nucleus pulposus mesenchymal stem cells (NPMSCs) have been identified in nucleus pulposus tissue and displayed great potential for the regeneration of IDD. However, biological differences between non-degenerative and degenerative nucleus pulposus-derived NPMSCs have remained to be defined. The aim of the present study was to compare the biological characteristics of human NPMSCs derived from non-degenerative and degenerative nucleus pulposus. NPMSCs were isolated from non-degenerative and degenerative nucleus pulposus, which were assessed using the Pfirrmann grading system. The biological characteristics of the NPMSCs, including the expression of surface markers, multipotent differentiation, colony formation, chemotactic cell migration, cell activity and stemness gene expression were compared. It was found that NPMSCs could be obtained from non-degenerative and degenerative human nucleus pulposus. However, degenerative nucleus pulposus-derived NPMSCs displayed decreased ability of colony formation, chemotactic migration, cell activity and expression of stemness genes compared with non-degenerative nucleus pulposus-derived NPMSCs. Therefore, NPMSCs derived from non-degenerative and degenerative nucleus pulposus show different biological behaviors. The degenerative status of
\end{abstract}

Correspondence to: Professor Dike Ruan or Professor Deli Wang, Department of Orthopaedics, Navy General Hospital, 6 Fucheng Road, Beijing 100048, P.R. China

E-mail: ruandikengh@163.com

E-mail:wangdelinavy@163.com

${ }^{*}$ Contributed equally

Key words: nucleus pulposus mesenchymal stem cells, intervertebral disc degeneration, cell therapy nucleus pulposus tissue should be considered when selecting NPMSCs as a source for clinical application.

\section{Introduction}

Lower back and neck pain are the major causes of disability and impose a huge economic burden on health care systems $(1,2)$. In the USA, expenditures for patients with spine problems were $\$ 85.9$ billion in 2005 , higher than those for numerous other causes of disability, including arthritis, and potentially fatal diseases such as cancer, while the prevalence remains to increase rapidly (2). Intervertebral disc degeneration (IDD) is the major cause of lower back and neck pain $(3,4)$. The normal intervertebral disc (IVD) is composed of three distinct components: The central hyper-hydrated nucleus pulposus, the outer annulus fibrosis, and the upper and lower cartilaginous endplates (4). Although the causes of IDD remain to be largely elusive, a decrease in the function and number of nucleus pulposus cells (NPCs) is known to be an initial trigger of IDD $(5,6)$. Various operative methods, including spinal fusion and artificial disc replacement, have been used to treat degenerative disc diseases, and have shown satisfactory results in alleviating pain. However, they are associated with certain complications, including prosthetic disc migration, extrusion or failure as well as accelerated degeneration of the IVDs adjacent to the fusion $(7,8)$. The most important disadvantage of these treatments is that they are aimed to alleviate the symptoms of IDD, but do not target the underlying disease itself. None of them aims to restore the biological function of the disc, or to retard or reverse $\operatorname{IDD}(3,9)$.

Cell therapy using mesenchymal stem cells (MSCs) has provided promising procedures for treating IDD in the field of regenerative medicine (3). Numerous stem cell types have been used in the field of cell transplantation for the treatment of IDD, such as bone marrow-derived stem cells (BMSCs) (10), adipose-derived MSCs (ADMSCs) (11), human umbilical tissue-derived cells (12) and synovium-derived stem cells (13). To successfully regenerate disks affected by IDD through cell therapy, stem cells must survive and function in 
the harsh IVD microenvironment, which is characterized by high osmolarity, limited nutrition, acidic $\mathrm{pH}$ and low oxygen tension $(14,15)$. Certain studies have reported that these stem cells lack viability, proliferation and matrix biosynthesis under conditions resembling the IVD microenvironment, which negatively influences the biological and metabolic vitality of stem cells and impairs their repair potential $(16,17)$.

In recent years, increasing evidence supported the existence of nucleus pulposus MSCs (NPMSCs) in the nucleus pulposus (18-24). They possess a similar capacity of chondrogenic differentiation to that of BMSCs (18) and have better adaptability to the harsh IVD microenvironment than ADMSCs (24). This suggests that NPMSCs may be a good candidate cell for IVD regeneration by cell therapy. Previous studies have proved that NPMSCs can be isolated from normal and degenerative nucleus pulposus (18-24). However, the biological properties of stem cells are crucial for the regenerative effects of cell therapy. To the best of our knowledge, whether non-degenerative (ND)-NPMSCs and degenerative (D)-NPMSCs have different biological characteristics has not been clarified. The present study aimed to compare the biological characteristics of human NPMSCs derived from non-degenerative and degenerative nucleus pulposus.

\section{Materials and methods}

Sample collection and group allocation. All procedures in the present study were approved by the Ethical Committee of Navy General Hospital (Beijing, China) and informed consent was obtained from all donors. All of the NP samples were obtained from 12 patients who underwent routine surgery for lumbar disorders between January and April 2015 in the Department of Orthopaedics, Navy General Hospital (Beijing, China). Samples were allocated to non-degenerative group (Pfirrmann grade I and II) and degenerative group (Pfirrmann grade IV and V) according to the Pfirrmann grading system (25). Therein, samples from 4 patients with congenital scoliosis and 2 patients with lumbar burst fracture were assigned to the non-degenerative group (NDG) and samples from 6 patients with lumbar intervertebral disc degeneration were assigned to degenerative group (DG). All of the assays described below were based on these two groups (NDG and DG).

Isolation and culture of NPMSCs. Tissue samples from the NDG and DG were washed 3 times with PBS and mechanically fragmented into $1 \mathrm{~mm}^{3}$ sections with ophthalmic scissor. Anulus fibrous, endplates, vessels and ligaments surrounding the NP were removed as much as possible. Subsequently, tissue fragments were digested with $0.2 \%(\mathrm{~m} / \mathrm{v})$ collagenase II (Sigma-Aldrich; Merck KGaG, Darmstadt, Germany) for $6 \mathrm{~h}$ at $37^{\circ} \mathrm{C}$. Primary cells were obtained after filtering through cell strainers with a pore size of $70 \mu \mathrm{m}$, resuspended in Dulbecco's modified Eagle's medium-low glucose (DMEM-LG) supplemented with $10 \%$ fetal bovine serum (FBS) (both from Sigma-Aldrich; Merck $\mathrm{KGaG}$ ), and were then seeded in $25 \mathrm{~cm}^{2}$ flasks at a density of $1 \times 10^{5} / \mathrm{ml}$. Cells were cultured in an incubator at $37^{\circ} \mathrm{C}$ in a humidified atmosphere containing $5 \% \mathrm{CO}_{2}$ and passaged upon reaching $80 \%$ confluence. The medium was changed every 3 days and the morphology of NPMSCs from different NP samples was observed under an inverted microscope. For further study, NPMSCs at passage 3 were resuspended in DMEM at the density required and subjected to various assays.

Flow cytometric analysis. Flow cytometric analysis was performed with an FC 500 flow cytometry instrument (Beckman Coulter, Brea, CA, USA). According to the standards proposed by the International Society For Cellular Therapy (ISCT), cell surface markers including CD90, CD105, CD73, human leukocyte antigen-antigen D related (HLA-DR), CD45, CD34 were used for identification of the MSC as phenotype in the present study (26). Of these, CD90, CD105, and CD 73 are highly expressed in MSCs, while HLA-DR, CD45 and CD34 are highly expressed in hematopoietic stem cells. In brief, $2 \times 10^{5}$ NPMSCs from each group were stained with phycoerythrin-conjugated monoclonal antibodies. The antibodies used are as follows: Mouse anti-CD90 antibody (ab92574, 1:200); mouse anti-CD105 antibody (ab11414, 1:200); Mouse anti-CD73 antibody (ab54217, 1:200); mouse anti-HLA-DR antibody (ab766, 1:200); mouse anti-CD45antibody (ab8216, 1:200); mouse anti-CD34 antibody (ab8536, 1:200) all supplied by Abcam (Cambridge, MA, USA). The cells were then incubated in the dark for $30 \mathrm{~min}$ at room temperature and washed with PBS, followed by being resuspended in $0.5 \mathrm{ml} \mathrm{PBS}$ and finally assessed by flow cytometry.

Multipotent differentiation. NPMSCs from various groups were induced to differentiate toward osteogenic, adipogenic and chondrogenic linages. All of the procedures were in accordance with the instructions provided by the manufacturers of the differentiation media.

Osteogenic differentiation. NPMSCs at passage 3 were resuspended at the density of $5 \times 10^{3} / \mathrm{ml}$ and seeded in 6-well plates. Osteogenic medium (Cyanogen, Palo Alto, CA, USA) was added when cells reached $60 \%$ confluence and changed every 3 days. After induction for 28 days, culture medium was removed and cells were fixed with $4 \%$ paraformaldehyde for $15 \mathrm{~min}$. Fixed MSCs were then stained with alizarin red (Sigma-Aldrich; Merck KGaG) and observed under an inverted microscope.

Adipogenic differentiation. NPMSCs at passage 3 were seeded in 6-well plates at a density of $2 \times 10^{3} / \mathrm{ml}$. Adipogenic medium A (Cyanogen) was added when cells reached $95 \%$ confluence and was replaced by Adipogenic medium B (Cyanogen) after 3 days. After $24 \mathrm{~h}$, adipogenic medium B was replaced by adipogenic medium A, and this cycle was repeated 3 times. The adipogenic induction was then maintained by incubation in adipogenic medium A. Oil red-O staining (Sigma-Aldrich; Merck $\mathrm{KGaG}$ ) was performed after induction for 28 days.

Chondrogenic differentiation. NPMSCs at passage 3 were resuspended in complete DMEM at a density of $5 \times 10^{5} / \mathrm{ml}$. To obtain a cell pellet, $\sim 0.5 \mathrm{ml}$ cell suspension (containing $2.5 \times 10^{5}$ cells) was pipetted into a $15-\mathrm{ml}$ centrifuge tube and centrifuged at $600 \times \mathrm{g}$ for $5 \mathrm{~min}$. The supernatant was then removed and $1 \mathrm{ml}$ chondrogenic medium (Cyanogen) was gently added to the tube without agitation. After culture for 28 days, cell pellets were fixed with $4 \%$ paraformaldehyde for 
Table I. Sequences of primers used for polymerase chain reaction.

\begin{tabular}{lll}
\hline Target gene & \multicolumn{1}{c}{ Forward 5'-3' } & \multicolumn{1}{c}{ Reverse 5'-3' } \\
\hline GAPDH & GAAGGTCGGAGTCAACGG & GGAAGATGGTGATGGGATT \\
Oct4 & GTATTCAGCCAAACGACCATCT & GCTTCCTCCACCCACTTCT \\
Nanog & ACCCCGTTTCACTGTGTTAGC & GACGGCAGCCAAGGTTATTAAA
\end{tabular}

GAPDH, glyceraldehyde 3-phosphate dehydrogenase; Oct4, octamer-binding transcription factor 4.

30 min and stained with alcian blue (Sigma-Aldrich; Merck $\mathrm{KGaG})$.

Colony formation assay. Cells at the third passage $\left(1,000 / \mathrm{cm}^{2}\right)$ were seeded in a 6-well plate and cultured with DMEM supplemented with $10 \%$ FBS. After culture for 14 days, cells were fixed with $4 \%$ paraformaldehyde for $15 \mathrm{~min}$ and stained with $1 \%$ crystal violet. Following washing with triple distilled water for 3 times, colonies that consisted of $>30$ nucleated cells were counted under an inverted microscope.

Chemotactic migration assay. Chemotactic migration was evaluated by a standard Transwell assay. Cells $\left(2 \times 10^{4} / 100 \mu \mathrm{l}\right)$ were seeded into the upper chamber with semipermeable membrane (pore size, $5 \mu \mathrm{m}$ ). Subsequently, each of the lower wells of the 24-well plate was filled with complete DMEM. After incubation for $12 \mathrm{~h}$, cells remaining on the upper surface of the membrane was wiped off with a cotton swab and cells, which had migrated to the lower surface, were fixed with $4 \%$ paraformaldehyde for $15 \mathrm{~min}$ and stained with $1 \%$ crystal violet. The migrated cells were quantified as the cell number per field of view.

Cell proliferative activity assay. Cell proliferative activity was assessed by a Cell Counting Kit-8 (CCK-8). In brief, cells at passage 3 were resuspended at a density of $5 \times 10^{4} / \mathrm{ml}$ and $100 \mu 1$ cell suspension was added into each well of 96 -well plate. At designated time-points ( $4 \mathrm{~h}, 1,3,5,7,9,11$ and 13 days), $10 \mu 1$ CCK-8 solution was added into the 96 -well plate and incubated at $37^{\circ} \mathrm{C}$ for $2 \mathrm{~h}$. The optical density (OD) value was analyzed using a spectrophotometer (UV762; Yoke Instrument, Co., Ltd., Shanghai, China). For each time-point, all experiments were performed three times in triplicate.

Reverse-transcription quantitative polymerase chain reaction (RT-qPCR) analysis of stemness genes. The expression of the stemness genes octamer-binding transcription factor 4 (Oct-4) and Nanog was determined by RT-qPCR. In brief total RNA was extracted using TRIzol reagent (Invitrogen; Thermo Fisher Scientific, Inc., MA, USA) according to the manufacturer's instructions. High Capacity cDNA Reverse Transcription kit (Thermo Fisher Scientific, Inc., MA, USA) was used to reverse transcribe RNA into cDNA. PCR was then performed following the manufacturer's instructions, as follows: $94^{\circ} \mathrm{C}$ for $60 \mathrm{sec}, 37^{\circ} \mathrm{C}$ for $60 \mathrm{sec}$ and $72^{\circ} \mathrm{C}$ for $2 \mathrm{~min}$ for 30 cycles. GADPH was used as the internal control; results were quantified as previously described (27). The sequences of the primers (Sanong Biotech Co., Ltd., Shanghai, China) are listed in Table I.
A

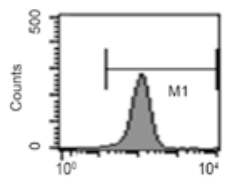

CD 105

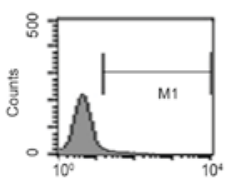

CD 45

B

D-NPMSCs

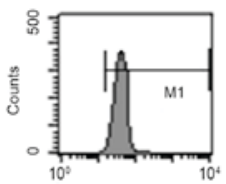

CD 105

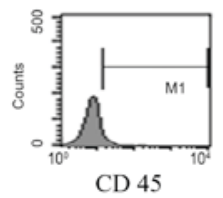

CD 45

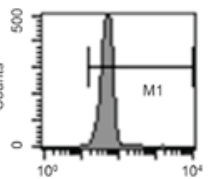

CD 90

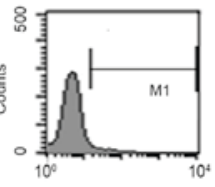

CD 34

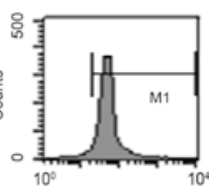

CD 90

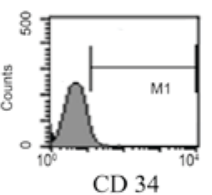

CD 34

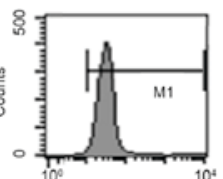

CD 73

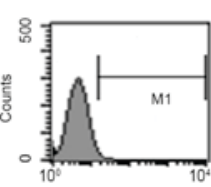

HLA-DR

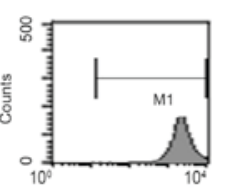

CD 73

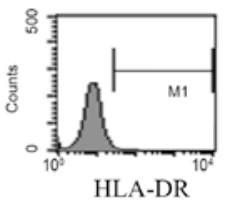

Figure 1. Surface molecule markers of second-passage NPMSCs. (A) On ND-NPMSCs, surface molecule markers (CD105, CD90 and CD73) were highly expressed, while the expression of hematopoietic stem cell markers (CD45, CD34 and HLA-DR) was low (all <4\%); (B) On D-NPMSCs, CD105, CD90 and CD73 were also highly expressed and hematopoietic stem cell marker expression was low (all <4\%). ND-NPMSCs, non-degenerated nucleus pulposus mesenchymal stem cells; HLA-DR, human leukocyte antigen-antigen D related.

Statistical analysis. SPSS 19.0 software (IBM, Armonk, NY, USA) was used for statistical analysis. Values are expressed as the mean \pm standard deviation. Statistical analysis was performed using analysis of variance. $\mathrm{P}<0.05$ was considered to indicate a statistically significant difference.

\section{Results}

Stem cell marker expression on D- and ND-NPMSCs. NPMSCs from the NDG and DG highly expressed CD105, CD90 and CD73 (characteristic cell surface marker of MSCs) and did not express CD45, CD34 and HLA-DR (characteristic cell surface markers of hematopoietic stem cells). In the NDG and DG, the positive expression rate for CD105, CD90 and CD73 was $>95 \%$ for all, respectively, and the positive expression rate for CD45, CD34 and HLA-DR was <4\% (Fig. 1). 


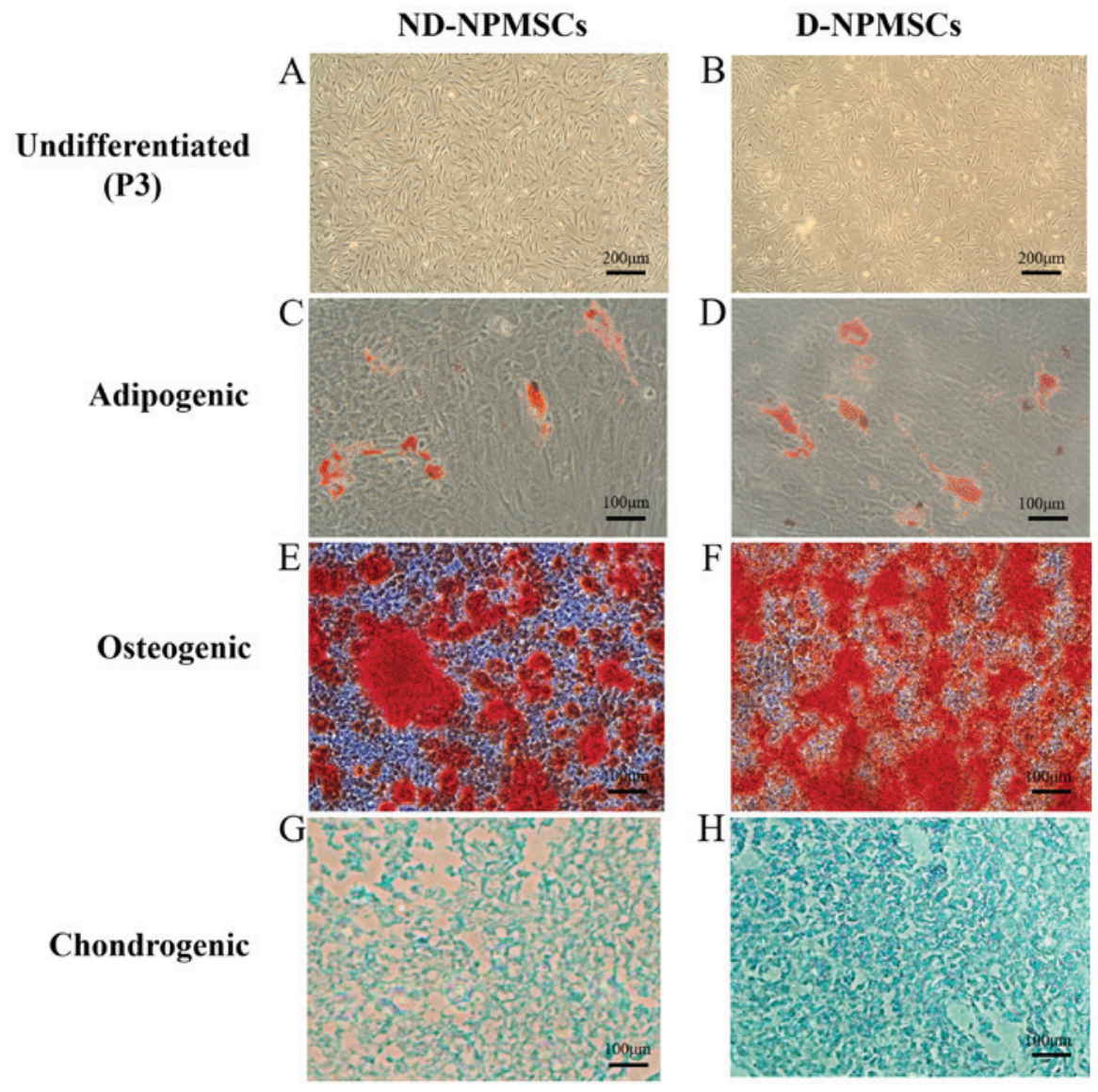

Figure 2. Cellular morphology and multipotent differentiation of NPMSCs (A and B). At passage 3, undifferentiated ND-NPMSCs and D-NPMSCs displayed elongated and spindle-like shapes; Scale bar, $200 \mu \mathrm{m}$. (C and D) After adipogenic induction for 28 days, ND-NPMSCs and D-NPMSCs were positive for Oil red-O staining and contained an abundance of lipid droplets; Scale bar, $100 \mu \mathrm{m}$. (E and F) After osteogenic induction for 28 days, ND-NPMSCs and D-NPMSCs were positive for alizarin red staining; Scale bar, $100 \mu \mathrm{m}$. (G and H) After chondrogenic induction for 28 days, the pellet of ND-NPMSCs and D-NPMSCs was positive for alcian blue staining; Scale bar, $100 \mu \mathrm{m}$. ND-NPMSCs, non-degenerated nucleus pulposus mesenchymal stem cells.

A

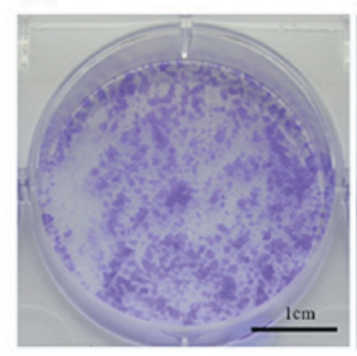

$\mathrm{D}$

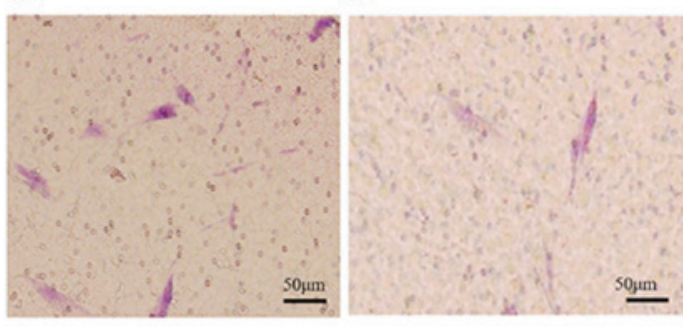

B

$\mathrm{E}$
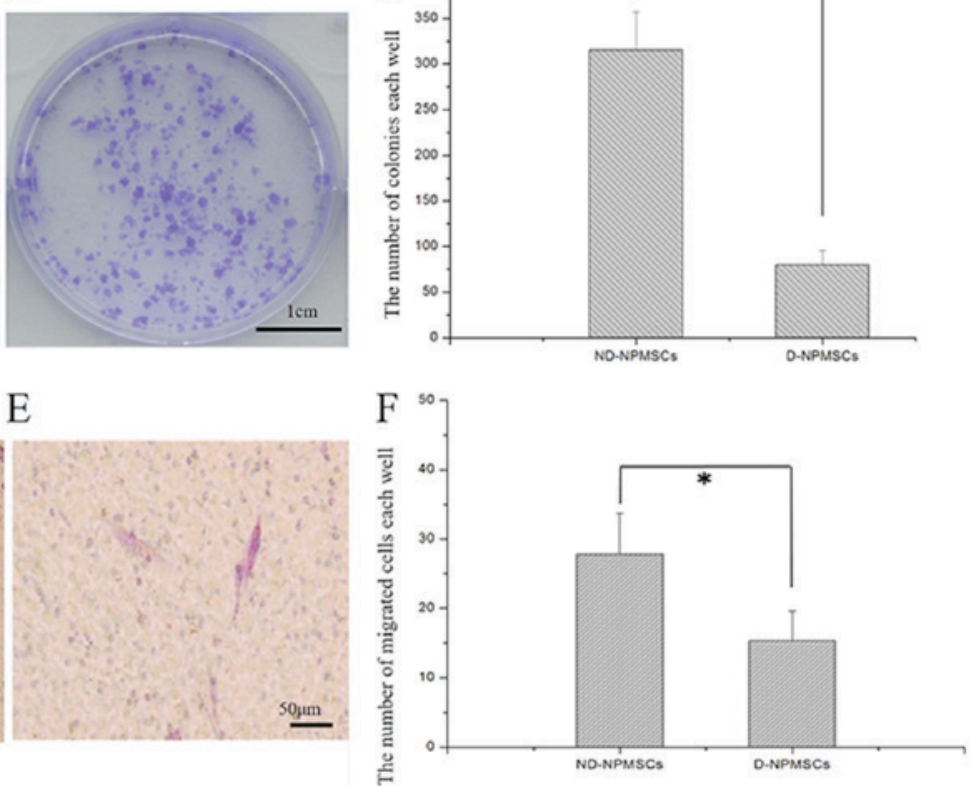

Figure 3. Colony formation and cell migration of NPMSCs. (A-C) Colony formation assay. (A) ND-NPMSCs and (B) D-NPMSCs were stained with crystal violet. (C) The number of colonies ( $>30$ cells) in the ND-NPMSC group $(315.5 \pm 41.87, \mathrm{n}=6$ ) was greater than that in the D-NPMSC group $(79.83 \pm 15.72, \mathrm{n}=6)$. (D-F) Assessment of cell migration via Transwell assay. Migrated cells in the (D) ND-NPMSC and (E) D-NPMSC groups were stained with crystal violet; Scale bar, $50 \mu \mathrm{m}$. (F) The number of migrated cells in the ND-NPMSC group $(27.41 \pm 6.58, \mathrm{n}=6)$ was higher than that in the D-NPMSC group (15.09 \pm 5.08 , $\mathrm{n}=6$ ). $\mathrm{P}<0.05$. Data is presented as mean \pm standard deviation. ND-NPMSCs, non-degenerated nucleus pulposus mesenchymal stem cells. 
$D$ - and ND-NPMSCs have a comparable multipotent differentiation capacity. At passage 3, undifferentiated ND-NPMSCs and D-NPMSCs displayed elongated and spindle-like shapes (Fig. 2A and B). Alizarin red staining showed deposition of large amounts of calcium nodules in ND-NPMSCs and D-NPMSCs (Fig. 2C and D). A large amount of lipid droplets deposited in cells subjected to Oil red-O staining indicated that ND-NPMSCs and D-NPMSCs differentiate into adipocytes after culture in adipogenic medium for 28 days (Fig. 2E and F). Chondrogenic differentiation was assessed by alcian blue staining after culture of cell pellets with chondrogenic medium. ND-NPMSCs and D-NPMSCs were positive for alcian blue staining (Fig. $2 \mathrm{G}$ and H). These results demonstrated that ND-NPMSCs and D-NPMSCs have a comparable capacity for multipotent differentiation.

ND-NPMSCs have a higher clonogenic and migratory capacity than D-NPMSCs. The clonogenicity of NPMSCs was evaluated after culture for 14 days (Fig. 3A-C). The number of colonies in each well of a 6 -well plate was $315.5 \pm 41.87$ in the ND-NPMSC and $79.83 \pm 15.72$ in the D-NPMSC group, and the difference between the two groups was significant $(\mathrm{P}<0.05)$. A Transwell assay was used to evaluate the capacity of NPMSCs for chemotactic migration, showing that the number of ND-NPMSCs migrated to the lower from the upper surface was nearly two times that of D-NPMSCs $(\mathrm{P}<0.05$; Fig. 3D-F).

ND-NPMSCs have a higher proliferative rate than $D$-NPMSCs. The proliferative activity of NPMSCs was assessed using aCCK- 8 assay. The results demonstrated that the cell proliferative activity of ND-NPMSCs and D-NPMSCs was not significantly different from $4 \mathrm{~h}$ to 3 days $(\mathrm{P}>0.05)$. However, the proliferative activity of ND-NPMSCs was higher than that of D-NPMSCs from days 5-13 ( $\mathrm{P}<0.05$; Fig. 4).

ND-NPMSCs show a higher expression of stemness genes than D-NPMSCs. The results of the RT-qPCR analysis showed that in ND-NPMSCs, the gene expression levels of Oct4 and Nanog were higher than those in D-NPMSCs $(\mathrm{P}<0.05)$. The relative expression of Oct4 and Nanog in ND-NPMSCs was $4.63 \pm 1.17$ and $7.36 \pm 1.19$ times that in D-NPMSCs, respectively (P<0.05; Fig. 5).

\section{Discussion}

MSCs are attractive sources of stem cells for regenerative medicine, possessing self-renewal and multi-lineage differentiation potentials (28). MSCs have been successfully isolated from numerous adult tissues (28), including bone marrow (10), adipose tissue (11), umbilical tissue (12) and synovium (13). NPMSCs have been isolated from nucleus pulposus and provide a promising approach for the treatment of IDD. In 2007, Risbud et al (29) isolated stem cells from the degenerative intervertebral disc, which were characterized by MSCs surface marker analysis and displayed osteogenic and adipogenic differentiation capacity. Therefore, these cells were defined as resident skeletal progenitor cells in the IVD. Subsequently, Brisby et al (30) identified progenitor cells inside the IVD by immunohistochemical staining. In addition, Blanco et al (18)

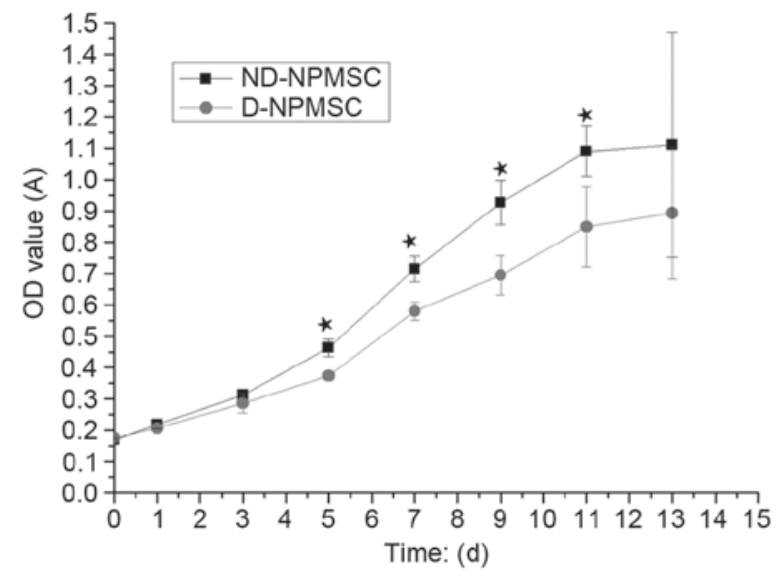

Figure 4. Cell viability according to a Cell Counting Kit- 8 assay. The OD value in ND-NPMSC group (day 5: 0.47 \pm 0.04 ; day 7: $0.72 \pm 0.05$; day 9; $0.92 \pm 0.08$; day 11: $1.08 \pm 0.09$; day 13: $1.12 \pm 0.35 ; \mathrm{n}=3$ ) was higher than that in the D-NPMSC group (day 5: 0.38 \pm 0.04 ; day 7: 0.58 \pm 0.04 ; day 9: 0.70 \pm 0.06 ; day 11: $0.85 \pm 0.13$; day 13: $0.89 \pm 0.23 ; \mathrm{n}=3)$ from day $5-13\left({ }^{\star} \mathrm{P}<0.05\right)$. Data is presented as mean \pm standard deviation. OD, optical density; d, days; A, absorbance; ND-NPMSCs, non-degenerated nucleus pulposus mesenchymal stem cells.

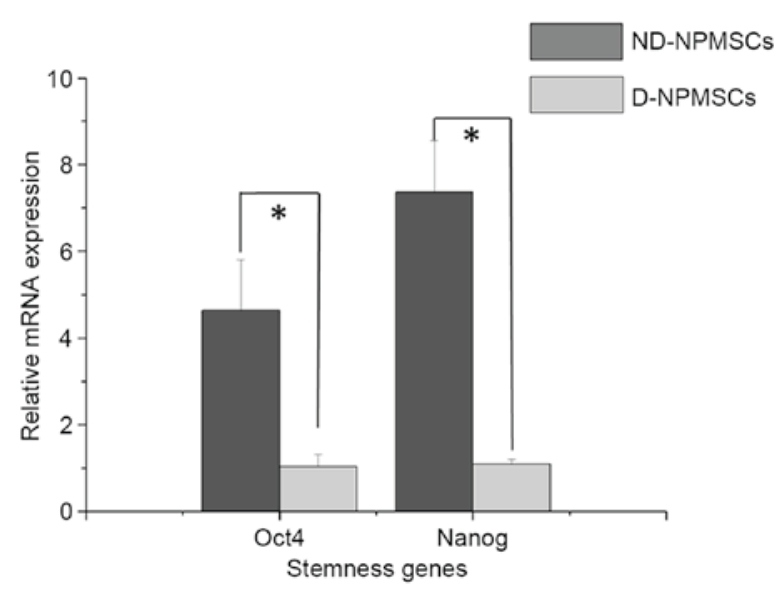

Figure 5. Comparison of relative gene expression of Oct4 and Nanog in ND-NPMSCs and D-NPMSCs. Gene expression levels of Oct4 in ND-NPMSCs $(4.68 \pm 1.17, \mathrm{n}=3)$ were higher than those in D-NPMSCs $(1.00 \pm 0.16, n=3)$. Gene expression levels of Nanog in ND-NPMSCs $(7.32 \pm 1.22, n=3)$ were higher than those in D-NPMSCs $(1.00 \pm 0.07, n=3)$. Gene expression was normalized to GAPDH. ${ }^{*} \mathrm{P}<0.05$. Data is presented as mean \pm standard deviation. ND-NPMSCs, non-degenerated nucleus pulposus mesenchymal stem cells; Oct4, octamer-binding transcription factor 4.

successfully isolated nucleus pulposus progenitor cells by using standard MSC culture medium, demonstrated their multilineage differentiation potential and identified their immunophenotype, which were all characteristics of MSCs. Based on the minimal criteria for defining MSCs proposed by ISCT, Blanco et al (18) defined these multipotent cells as NPMSCs.

In the present study, the same method (18) was used to isolate and culture cells with the same characteristics from 6 non-degenerative and 6 degenerative nucleus pulposus tissues. NPMSCs in each of the two groups attached well and the positive rate of CD105, CD90 and CD73 surpassed 95\% while that of CD45, CD34 and HLA-DR was $>4 \%$. ND-NPMSCs and D-NPMSCs had multilineage differentiation potential toward osteogenesis, chondrogenesis and adipogenesis. All of these 
results confirmed the feasibility of the isolation and culture NPMSCs from non-degenerative and degenerative nucleus pulposus.

Along with aging and accumulation of noxious stimuli, the intervertebral disc is gradually degenerated due to the reduction of activity as well as the amount and production of extracellular matrix by inherent cells $(31,32)$. However, few studies have compared the biological characteristics of NPMSCs isolated from human non-degenerative and degenerative nucleus pulposus tissues. In the present study, clonogenic and migratory capacity were performed, and results indicated that ND-NPMSCs exhibited better capacities, compared with D-NPMSCs. Furthermore, a CCK-8 assay was used to compare cell proliferative activity of ND-NPMSCs and D-NPMSCs. At 5, 7, 9, 11 and 13 days after cell seeding, the OD value of D-NPMSCs was obviously higher than that of D-NPMSCs, which directly reflected that the cell proliferative activity of ND-NPMSCs was significantly higher than that of D-NPMSCs. It was conjectured that this difference maybe attributed to the accumulated injury in the disc with long-term stimulus of stress and inflammatory cytokines in the process of degeneration.

MSCs possess the capacity of self-renewal and multilineage differentiation (33). Numerous studies have found that the capacity of proliferation and differentiation of adult stem cells decreased with age and degeneration, which may result from lessened expression of genes regulating the maintenance of stemness of stem cells (34-37). Furthermore, expression of stemness genes has an important role in the regulation of multilineage differentiation of stem cells and decreases with cell differentiation (38-40). Tsai et al (41) also found that Oct4 and Nanog genes were highly expressed in adult MSCs, and that proliferation and differentiation of MSCs decreased after knockout of the Oct4 and Nanog genes. In the present study, RT-qPCR analysis showed that relative expression levels of Oct 4 and Nanog in the NDG were 4.63 and 7.36 times those in the DG. These results suggested that ND-NPMSCs displayed better self-renewal and multilineage differentiation capacity than D-NPMSCs. The adverse stimulus in the process of degeneration may be the major reason for these findings.

In conclusion, the present study demonstrated that NPMSCs isolated from non-degenerative nucleus pulposus tissue exhibited better in stem cell marker expression, clonogenic and migratory capacity, proliferative rate and expression of stemness genes, compared with NPMSCs isolated from degenerative nucleus pulposus tissue. Therefore, the degenerative status of nucleus pulposus tissue should be considered when selecting a source of NPMSCs for clinical application.

\section{Acknowledgements}

This study was supported by the National Natural Science Foundation of China (nos. 81171740 and 81470102).

\section{References}

1. Vos T, Flaxman AD, Naghavi M, Lozano R, Michaud C, Ezzati M, Shibuya K, Salomon JA, Abdalla S, Aboyans V, et al: Years lived with disability (YLDs) for 1160 sequelae of 289 diseases and injuries 1990-2010: A systematic analysis for the Global Burden of Disease Study 2010. Lancet 380: 2163-2196, 2012.
2. Martin BI, Deyo RA, Mirza SK, Turner JA, Comstock BA, Hollingworth W and Sullivan SD: Expenditures and health status among adults with back and neck problems. JAMA 299: 656-664, 2008

3. Sakai D and Andersson GB: Stem cell therapy for intervertebral disc regeneration: Obstacles and solutions. Nat Rev Rheumatol 11: 243-256, 2015.

4. Adams MA and Roughley PJ: What is intervertebral disc degeneration and what causes it? Spine (Phila Pa 1976) 31: 2151-2161, 2006.

5. Buckwalter JA: Aging and degeneration of the human intervertebral disc. Spine (Phila Pa 1976) 20: 1307-1314, 1995

6. Roughley PJ: Biology of intervertebral disc aging and degeneration: Involvement of the extracellular matrix. Spine (Phila Pa 1976) 29: 2691-2699, 2004.

7. Lund T and Oxland TR: Adjacent level disk disease-is it really a fusion disease? Orthop Clin North Am 42: 529-541, viii, 2011.

8. Errico TJ: Lumbar disc arthroplasty. Clin Orthop Relat Res: 106-117, 2005.

9. Hohaus C, Ganey TM, Minkus Y and Meisel HJ: Cell transplantation in lumbar spine disc degeneration disease. Eur Spine J 17 (Suppl 4): S492-S503, 2008.

10. Wei A, Tao H, Chung SA, Brisby H, Ma DD and Diwan AD: The fate of transplanted xenogeneic bone marrow-derived stem cells in rat intervertebral discs. J Orthop Res 27: 374-379, 2009.

11. Chun HJ, Kim YS, Kim BK, Kim EH, Kim JH, Do BR, Hwang SJ, Hwang JY and Lee YK: Transplantation of human adipose-derived stem cells in a rabbit model of traumatic degeneration of lumbar discs. World Neurosurg 78: 364-371, 2012.

12. Leckie SK, Sowa GA, Bechara BP, Hartman RA, Coelho JP, Witt WT, Dong QD, Bowman BW, Bell KM, Vo NV, et al: Injection of human umbilical tissue-derived cells into the nucleus pulposus alters the course of intervertebral disc degeneration in vivo. Spine J 13: 263-272, 2013.

13. Miyamoto T, Muneta T, Tabuchi T, Matsumoto K, Saito H, Tsuji K and Sekiya I: Intradiscal transplantation of synovial mesenchymal stem cells prevents intervertebral disc degeneration through suppression of matrix metalloproteinase-related genes in nucleus pulposus cells in rabbits. Arthritis Res Ther 12: R206, 2010.

14. Urban JP: The role of the physicochemical environment in determining disc cell behaviour. Biochem Soc Trans 30: 858-864, 2002.

15. Holm S and Nachemson A: Variations in the nutrition of the canine intervertebral disc induced by motion. Spine (Phila Pa 1976) 8: 866-874, 1983.

16. Wuertz K, Godburn K, Neidlinger-Wilke C, Urban J and Iatridis JC: Behavior of mesenchymal stem cells in the chemical microenvironment of the intervertebral disc. Spine (Phila Pa 1976) 33: 1843-1849, 2008.

17. Liang C, Li H, Tao Y, Zhou X, Li F, Chen G and Chen Q: Responses of human adipose-derived mesenchymal stem cells to chemical microenvironment of the intervertebral disc. J Transl Med 10: 49, 2012.

18. Blanco JF, Graciani IF, Sanchez-Guijo FM, Muntión S, Hernandez-Campo P, Santamaria C, Carrancio S, Barbado MV, Cruz G, Gutierrez-Cosío S, et al: Isolation and characterization of mesenchymal stromal cells from human degenerated nucleus pulposus: Comparison with bone marrow mesenchymal stromal cells from the same subjects. Spine (Phila Pa 1976) 35: 2259-2265, 2010

19. Tao YQ, Liang CZ, Li H, Zhang YJ, Li FC, Chen G and Chen QX: Potential of co-culture of nucleus pulposus mesenchymal stem cells and nucleus pulposus cells in hyperosmotic microenvironment for intervertebral disc regeneration. Cell Biol Int 37: 826-834, 2013.

20. Erwin WM, Islam D, Eftekarpour E, Inman RD, Karim MZ and Fehlings MG: Intervertebral disc-derived stem cells: Implications for regenerative medicine and neural repair. Spine (Phila Pa 1976) 38: 211-216, 2013.

21. Guan X, Ma X, Zhang L, Feng H and Ma Z: Evaluation of CD24 as a marker to rapidly define the mesenchymal stem cell phenotype and its differentiation in human nucleus pulposus. Chin Med J (Engl) 127: 1474-1481, 2014.

22. Li H, Tao Y, Liang C, Han B, Li F, Chen G and Chen Q: Influence of hypoxia in the intervertebral disc on the biological behaviors of rat adipose- and nucleus pulposus-derived mesenchymal stem cells. Cells Tissues Organs 198: 266-277, 2013. 
23. Zhang H, Ma X, Zhang L, Guan X, Bai T and Xue C: The ability to form cartilage of NPMSC and BMSC in SD rats. Int J Clin Exp Med 8: 4989-4996, 2015.

24. Han B, Wang HC, Li H, Tao YQ, Liang CZ, Li FC, Chen G and Chen QX: Nucleus pulposus mesenchymal stem cells in acidic conditions mimicking degenerative intervertebral discs give better performance than adipose tissue-derived mesenchymal stem cells. Cells Tissues Organs 199: 342-352, 2014.

25. Pfirrmann CW, Metzdorf A, Zanetti M, Hodler J and Boos N Magnetic resonance classification of lumbar intervertebral disc degeneration. Spine (Phila Pa 1976) 26: 1873-1878, 2001.

26. Dominici M, Le Blanc K, Mueller I, Slaper-Cortenbach I, Marini F, Krause D, Deans R, Keating A, Prockop Dj and Horwitz E: Minimal criteria for defining multipotent mesenchymal stromal cells. The international society for cellular therapy position statement. Cytotherapy 8: 315-317, 2006.

27. Livak KJ and Schmittgen TD: Analysis of relative gene expression data using real-time quantitative PCR and the 2(-Delta Delta C(T)) method. Methods 25: 402-408, 2001

28. Pittenger MF, Mackay AM, Beck SC, Jaiswal RK, Douglas R, Mosca JD, Moorman MA, Simonetti DW, Craig S and Marshak DR: Multilineage potential of adult human mesenchymal stem cells. Science 284: 143-147, 1999.

29. Risbud MV, Guttapalli A, Tsai TT, Lee JY, Danielson KG Vaccaro AR, Albert TJ, Gazit Z, Gazit D and Shapiro IM: Evidence for skeletal progenitor cells in the degenerate human intervertebral disc. Spine (Phila Pa 1976) 32: 2537-2544, 2007.

30. Brisby H, Papadimitriou N, Brantsing C, Bergh P, Lindahl A and Barreto Henriksson $\mathrm{H}$ : The presence of local mesenchymal progenitor cells in human degenerated intervertebral discs and possibilities to influence these in vitro: A descriptive study in humans. Stem Cells Dev 22: 804-814, 2013.

31. Ariga K, Miyamoto S, Nakase T, Okuda S, Meng W, Yonenobu K and Yoshikawa $\mathrm{H}$ : The relationship between apoptosis of endplate chondrocytes and aging and degeneration of the intervertebral disc. Spine (Phila Pa 1976) 26: 2414-2420, 2001

32. Liebscher T, Haefeli M, Wuertz K, Nerlich AG and Boos N: Age-related variation in cell density of human lumbar intervertebral disc. Spine (Phila Pa 1976) 36: 153-159, 2011.
33. Sensebé L and Fleury-Cappellesso S: Biodistribution of mesenchymal stem/stromal cells in a preclinical setting. Stem Cells Int 2013: 678063, 2013.

34. Sharpless NE and DePinho RA: How stem cells age and why this makes us grow old. Nat Rev Mol Cell Biol 8: 703-713, 2007.

35. Chang HX, Yang L, Li Z, Chen G and Dai G: Age-related biological characterization of mesenchymal progenitor cells in human articular cartilage. Orthopedics 34: e382-e388, 2011.

36. Alt EU, Senst C, Murthy SN, Slakey DP, Dupin CL, Chaffin AE, Kadowitz PJ and Izadpanah R: Aging alters tissue resident mesenchymal stem cell properties. Stem Cell Res 8: 215-225, 2012.

37. Collins-Hooper H, Woolley TE, Dyson L, Patel A, Potter P, Baker RE, Gaffney EA, Maini PK, Dash PR and Patel K: Age-related changes in speed and mechanism of adult skeletal muscle stem cell migration. Stem Cells 30: 1182-1195, 2012.

38. Boyer LA, Lee TI, Cole MF, Johnstone SE, Levine SS, Zucker JP, Guenther MG, Kumar RM, Murray HL, Jenner RG, et al: Core transcriptional regulatory circuitry in human embryonic stem cells. Cell 122: 947-956, 2005.

39. Darr H, Mayshar Y and Benvenisty N: Overexpression of NANOG in human ES cells enables feeder-free growth while inducing primitive ectoderm features. Development 133: 1193-1201, 2006.

40. Barrand S and Collas P: Chromatin states of core pluripotency-associated genes in pluripotent, multipotent and differentiated cells. Biochem Biophys Res Commun 391: 762-767, 2010.

41. Tsai CC, Su PF, Huang YF, Yew TL and Hung SC: Oct4 and Nanog directly regulate Dnmtl to maintain self-renewal and undifferentiated state in mesenchymal stem cells. Mol Cell 47: 169-182, 2012. 